weeks on; 1 week off]). Eligible pts had aEC with 1 prior platinum-based chemotherapy regimen or up to 2 prior platinum-based chemotherapy regimens, if 1 was given in the neoadjuvant/adjuvant setting. Randomization was stratified by DNA mismatch repair (MMR) status (centrally determined); pts with proficient (p)MMR tumors were further stratified by ECOG PS, geographic region, and prior history of pelvic radiation. Primary endpoints were PFS by blinded independent central review per RECIST v1.1 and OS. Key secondary endpoints included objective response rate (ORR) and safety. A graphical approach for multiplicity to control for type 1 error was used to test PFS for pts with pMMR aEC, then pts irrespective of MMR tumor status (i.e., all comers), followed SGO 2021 LEN 309 Abstract

Results 827 Pts (pMMR, $\mathrm{n}=697$; dMMR, $\mathrm{n}=130$ ) were randomized to receive LEN + pembro $(n=411)$ or TPC $(\mathrm{n}=416)$. Median follow-up was 12.2 mo for pts randomized to LEN + pembro and 10.7 mo for pts randomized to TPC (data cutoff October 26, 2020). PFS was significantly improved with LEN + pembro vs TPC in pMMR aEC (median 6.6 vs $3.8 \mathrm{mo}$; HR 0.60) and in all-comers (median 7.2 vs $3.8 \mathrm{mo}$; HR 0.56). OS was significantly longer with LEN + pembro vs TPC in pMMR aEC (median 17.4 vs 12.0 mo; HR 0.68) and in all-comers (median 18.3 vs $11.4 \mathrm{mo}$; HR 0.62). ORR was significantly greater with LEN + pembro vs TPC in pMMR aEC (30.3\% vs $15.1 \%)$ and in all-comers $(31.9 \%$ vs $14.7 \%)$. Additional results are in the table. Median treatment duration was 231 days with LEN + pembro and 104.5 days with TPC. Overall, anygrade treatment-emergent adverse events (TEAEs) occurred at similar rates across treatment arms. Grade $\geq 3$ TEAEs occurred in $89 \%$ of pts with LEN + pembro and $73 \%$ of pts with TPC. In the LEN + pembro arm, $30.8 \%$ pts discontinued LEN, $18.7 \%$ discontinued pembro, and $14.0 \%$ discontinued both study treatments due to a TEAE; the most common TEAEs were hypertension (64\%), hypothyroidism (57\%), diarrhea (54\%) and nausea $(50 \%)$.

\begin{tabular}{|c|c|c|c|c|}
\hline \multicolumn{5}{|l|}{ Table } \\
\hline & \multicolumn{2}{|c|}{ PMMR } & \multicolumn{2}{|c|}{ All-comers } \\
\hline & $\begin{array}{l}\text { LEN + pembro } \\
(n=346)\end{array}$ & $\begin{array}{c}\text { TPC } \\
(N=351)\end{array}$ & $\begin{array}{l}\text { LEN + pembro } \\
(n=411)\end{array}$ & $\begin{array}{c}\text { TPC } \\
(N=416)\end{array}$ \\
\hline Median PFS, months $(95 \% \mathrm{CI})$ & $6.6(5.6,7.4)$ & $3.8(3.6,5.0)$ & $7.2(5.7,7.6)$ & $3.8(3.6,4.2)$ \\
\hline $\begin{array}{l}\text { PFS HR (95\% Cl) } \\
\text { P-value }\end{array}$ & \multicolumn{2}{|c|}{$\begin{array}{c}0.60(0.50,0.72) \\
<0.0001\end{array}$} & \multicolumn{2}{|c|}{$\begin{array}{c}0.56(0.47,0.66) \\
<0.0001\end{array}$} \\
\hline Median OS, months (95\% Cl) & $\begin{array}{c}17.4 \\
(14.2,19.9) \\
\end{array}$ & $\begin{array}{c}12.0 \\
(10.8,13.3)\end{array}$ & $\begin{array}{c}18.3 \\
(15.2,20.5)\end{array}$ & $\begin{array}{c}11.4 \\
(10.5,12.9)\end{array}$ \\
\hline $\begin{array}{l}\text { OS HR vs TPC }(95 \% \text { Cl) } \\
\text { P-value }\end{array}$ & \multicolumn{2}{|c|}{$\begin{array}{c}0.68(0.56,0.84) \\
0.0001\end{array}$} & \multicolumn{2}{|c|}{$\begin{array}{c}0.62(0.51,0.75) \\
<0.0001\end{array}$} \\
\hline ORR, \% (95\% CI) & $\begin{array}{c}30.3 \\
(25.5,35.5)\end{array}$ & $\begin{array}{c}15.1 \\
(11.5,19.3)\end{array}$ & $\begin{array}{c}31.9 \\
(27.4,36.6)\end{array}$ & $\begin{array}{c}14.7 \\
(11.4,18.4)\end{array}$ \\
\hline $\begin{array}{l}\text { ORR Difference, \% } \\
\text { P-value }\end{array}$ & \multicolumn{2}{|c|}{$\begin{array}{c}15.2 \\
<0.0001\end{array}$} & \multicolumn{2}{|c|}{$\begin{array}{c}17.2 \\
<0.0001\end{array}$} \\
\hline $\begin{array}{l}\text { Median duration of response, } \\
\text { months (range) }\end{array}$ & $\begin{array}{c}9.2 \\
\left(1.6^{*}, 23.7^{*}\right)\end{array}$ & $\begin{array}{c}5.7 \\
\left(0.0^{+}, 24.2^{4}\right)\end{array}$ & $\begin{array}{c}14.4 \\
\left(1.6^{*}, 23.7^{*}\right)\end{array}$ & $\begin{array}{c}5.7 \\
\left(0.0^{*}, 24.2^{*}\right)\end{array}$ \\
\hline
\end{tabular}

Conclusions LEN + pembro demonstrated statistically significant and clinically meaningful improvements in PFS, OS, and ORR vs TPC both in pts with aEC that was pMMR and in pts with aEC irrespective of MMR status. The safety profile of LEN + pembro was manageable and consistent with previously reported studies.
0009/\#786 RUCAPARIB VS CHEMOTHERAPY IN PATIENTS
WITH ADVANCED, RELAPSED OVARIAN CANCER
AND A DELETERIOUS BRCA MUTATION: EFFICACY
AND SAFETY FROM ARIEL4, A RANDOMIZED
PHASE 3 STUDY

${ }^{1} \mathrm{R}$ Kristeleit ${ }^{*},{ }^{2} \mathrm{~A}$ Lisyanskaya, ${ }^{3} \mathrm{~A}$ Fedenko, ${ }^{4} \mathrm{M}$ Dvorkin, ${ }^{5} \mathrm{AC}$ De Melo, ${ }^{6} \mathrm{Y}$ Shparyk, ${ }^{7}$ I Rakhmatullina, ${ }^{8}$ B Bondarenko, ${ }^{9} \mathrm{~N}$ Colombo, ${ }^{10} \mathrm{~V}$ Svintsitskiy, ${ }^{11} \mathrm{~L}$ Biela, ${ }^{12} \mathrm{M}$ Nechaeva, ${ }^{13} \mathrm{~F}$ Raspagliesi, ${ }^{14} \mathrm{G}$ Scambia, ${ }^{15} \mathrm{D}$ Cibula, ${ }^{16} \mathrm{R}$ Póka, ${ }^{17} \mathrm{~A}$ Oaknin, ${ }^{18} \mathrm{~T}$ Safra, ${ }^{19} \mathrm{~B}$ MackowiakMatejczyk, ${ }^{20} \mathrm{~L}$ Ma. ${ }^{1}$ Department of Oncology, Guy's and St Thomas' NHS Foundation Trust, Great Maze Pond, London, UK; ${ }^{2}$ St. Petersburg State Budgetary Institution of Healthcare, Department of Gynaecological Oncology, St. Petersburg, Russian Federation; ${ }^{3}$ Department of Chemotherapy, N.n. Blokhin Russian Cancer Research Center, Moscow, Russian Federation; ${ }^{4}$ Omsk Region Clinical Oncologic Dispensary, Tba, Omsk, Russian Federation; ${ }^{5}$ Brazilian National Cancer Institute, Division of Clinical Research, Rio de Janeiro, Brazil; ${ }^{6}$ Department of Chemotherapy, Lviv Regional Oncology Dispensary, Lviv, Ukraine; 'Department of Chemotherapy, Republic Clinical Oncology Dispensary of The Ministry of Healthcare of Republic of Bashkortostan, Ufa, Russian Federation; ${ }^{8}$ Dnipropetrovsk Medical Academy, Oncology and Medical Radiology Department, dnipro, Ukraine; ${ }^{9}$ University of Milan-Bicocca and European Institute of Oncology, Milan, Gynecologic Oncology, Milan, Italy; ${ }^{10}$ National Cancer Institute, Oncogynecology, Kiyv, Ukraine; ${ }^{11}$ Instituto de Oncologia do Parana (IOP), Clinical Research Center, Curitiba, Brazil; ${ }^{12}$ Arkhangelsk Clinical Oncological Dispensary, Department of Chemotherapy, Arkhangelsk, Russian Federation; ${ }^{13}$ Fondazione IRCCS Istituto nazionale Tumori - Milan, Surgery, Milan, Italy; ${ }^{14}$ Fondazione Policlinico Universitario A. Gemelli IRCCS and Scientific Directorate, Gynecologic Oncology Unit, Rome, Italy; ${ }^{15}$ General Faculty Hospital in Prague, First Faculty of Medicine, Charles University, Department of Obstetrics and Gynecology, Prague, Czech Republic; ${ }^{16} \mathrm{Clinical}$ Center, University of Debrecen, Department of Obstetrics and Gynecology, TBA, Hungary; ${ }^{17}$ Vall d'Hebron Institute of Oncology (VHIO), Hospital Universitari Vall d'Hebron, Medical Oncology, Barcelona, Spain; ${ }^{18}$ TLVMC, Gynecologic Oncology, Tel Aviv, Israel; ${ }^{19}$ Marii Sklodowskiej-Curie, Bialostockie Centrum Onkologii Im, Białystok, Poland; ${ }^{20} T B A$, Rocky Mountain Cancer Centers, Lakewood, USA

\subsection{6/ijgc-2021-IGCS.9}

Objectives Prospective studies comparing poly(adenosine diphosphate-ribose) polymerase (PARP) inhibitors with standard-of-care (SOC) chemotherapy (CT) in patients (pts) with relapsed ovarian cancer (OC) are currently limited. ARIEL4 (NCT02855944) is a phase 3, randomized, open-label, international, multicenter study of the efficacy and safety of rucaparib vs SOC CT as treatment for PARP-inhibitor naïve pts with relapsed high-grade epithelial ovarian, fallopian tube or primary peritoneal cancer, who had a deleterious BRCA1/2 (BRCA) mutation and had received $\geq 2$ prior CT regimens.

Methods Pts were randomized 2:1 to oral rucaparib $600 \mathrm{mg}$ twice daily or SOC CT and stratified based on progressionfree interval $(\geq 1$ to $<6$ months $=$ platinum resistant; $\geq 6$ to $<12$ months $=$ partially platinum sensitive; $\geq 12$ months $=$ fully platinum sensitive). Pts in the CT arm with platinumresistant or partially platinum-sensitive disease received weekly paclitaxel $60-80 \mathrm{mg} / \mathrm{m}^{2}$; pts with fully platinum-sensitive disease received investigator's choice of platinum-based CT (single-agent carboplatin or cisplatin, or platinum doublet [carboplatin + paclitaxel, carboplatin + gemcitabine, or cisplatin + gemcitabine]). Pre-study-treatment plasma samples were assessed for BRCA reversion mutations. The primary endpoint was investigator-assessed progression-free survival (PFS). Secondary endpoints included objective response rate (ORR) per RECIST and safety. Each efficacy endpoint was first evaluated in the efficacy population (randomized pts with deleterious BRCA mutations excluding those with BRCA reversion mutations), stepping down to the intent-to-treat (ITT) population (all randomized pts). 
Abstract 0009/\#786 Table 1

\begin{tabular}{|c|c|c|c|c|}
\hline \multirow[b]{2}{*}{$\begin{array}{l}\text { Efficacy endpoint/ } \\
\text { Statistical test }\end{array}$} & \multicolumn{2}{|c|}{ Efficacy population } & \multicolumn{2}{|c|}{ IIT population } \\
\hline & $\begin{array}{l}\text { Rucaparib } \\
(\mathrm{n}=220)\end{array}$ & $\underset{\substack{\text { CT } \\
(n=105)}}{ }$ & $\begin{array}{l}\text { Rucaparib } \\
\text { (n=233) }\end{array}$ & $\begin{array}{c}\mathrm{CT} \\
(\mathrm{n}=116)\end{array}$ \\
\hline $\begin{array}{l}\text { Median } \text { PFS }^{3}(95 \% \\
\text { Cl), months }\end{array}$ & $7.4(7.3-9.1)$ & $5.7(5.5-7.3)$ & $7.4(6.7-7.9)$ & $5.7(5.5-6.7)$ \\
\hline $\begin{array}{l}\text { Stratified Cox } \\
\text { proportional hazard } \\
\text { model }\end{array}$ & \multicolumn{2}{|c|}{$\begin{array}{c}\text { HR } 0.639(95 \% \mathrm{Cl} 0.489-0.835) ; \\
P=0.0010\end{array}$} & \multicolumn{2}{|c|}{$\begin{array}{c}\text { HR } 0.665(95 \% \mathrm{Cl} 0.516-0.858) ; \\
P=0.0017\end{array}$} \\
\hline $\begin{array}{l}\text { RECIST ORR }{ }^{\mathrm{b}}, \mathrm{n} / \mathrm{N}(\%) \\
{[95 \% \mathrm{CI}]}\end{array}$ & $\begin{array}{c}85 / 211(40.3) \\
{[33.6-47.2]}\end{array}$ & $\begin{array}{l}31 / 96(32.3) \\
{[23.1-42.6]}\end{array}$ & $\begin{array}{c}85 / 224(37.9) \\
{[31.6-44.7]}\end{array}$ & $\begin{array}{c}32 / 106(30.2) \\
{[21.7-39.9]}\end{array}$ \\
\hline $\begin{array}{l}\text { Stratified Cochran- } \\
\text { Mantel-Haenszel test }\end{array}$ & \multicolumn{2}{|c|}{$P=0.1287$} & \multicolumn{2}{|c|}{$P=0.1250$} \\
\hline
\end{tabular}

Primase

Pcimarcx endpoint; 'Secsondacx endpoint; only patients with measurable aisease were included in this analysis. Response Evaluation Criteria In Solid Tumors, version 1.1
Results 233 pts were randomized to rucaparib and 116 to CT (visit cutoff Sep 30, 2020); 179 (51.3\%) had platinum-resistant, $96(27.5 \%)$ had partially platinum-sensitive, and 74 $(21.2 \%)$ had fully platinum-sensitive disease. 23 pts (6.6\%) with BRCA reversion mutations and 1 pt without a BRCA mutation were excluded from the efficacy population. Median PFS was significantly longer with rucaparib vs CT in both the efficacy and ITT populations (Table). In an exploratory analysis of pts with BRCA reversion mutations, median PFS was shorter with rucaparib $(n=13)$ vs CT $(n=10) ; 2.9$ vs 5.5 months, hazard ratio 2.769 (95\% CI, 0.989-7.755). ORR was not significantly different between the rucaparib and CT arms in both populations (Table). Adverse events were consistent with the known safety profiles of rucaparib and CT.

Conclusions Patients with BRCA-mutated advanced, relapsed OC who received rucaparib had a significant improvement in PFS vs SOC CT. No new safety signals were identified. This is the first prospective report from a randomized trial demonstrating that the presence of a BRCA reversion mutation predicts for primary resistance to rucaparib.

\section{0/\#784 ADJUVANT CHEMOTHERAPY FOLLOWING CHEMO- RADIATION AS PRIMARY TREATMENT FOR LOCALLY ADVANCED CERVICAL CANCER COMPARED TO CHEMO-RADIATION ALONE: THE RANDOMISED PHASE 3 OUTBACK TRIAL}

K Moore*. TBA, Tba, TBA, USA

10.1136/ijgc-2021-IGCS.10

Background Cervical cancer is a common cause of cancerrelated death among women worldwide. Standard treatment for locally advanced disease is chemoradiation. However, a significant percentage of women still relapse and die from the development of distant metastatic disease. OUTBACK was designed to determine the effects of giving adjuvant chemotherapy after chemoradiation on survival.

Methods OUTBACK is an international randomized phase 3 trial of the Gynecologic Cancer InterGroup (GCIG). Participating groups (countries) included ANZGOG (Australia and New Zealand), NRG (USA, Saudi Arabia, Canada, China), and Singapore. Eligible women had locally advanced cervical cancer (FIGO 2008 stage IB1 \& node positive, IB2, II, IIIB or IVA) that was suitable for primary treatment with chemoradiation with curative intent. Women were randomly assigned to either standard cisplatin-based chemo-radiation (control) or standard cisplatin-based chemo-radiation followed by adjuvant chemotherapy (ACT) with 4 cycles of carboplatin and paclitaxel, after stratification for nodal status, participating site, FIGO stage, age, and planned extendedfield radiotherapy. The primary end point was overall survival (OS) at 5 years. Secondary endpoints included progression-free survival (PFS); adverse events (AE); and patterns of disease recurrence. The target sample size of 900 provided $80 \%$ power with $95 \%$ confidence to detect an improvement in OS at 5 years from $72 \%$ (control) to $80 \%$ (ACT), with some over-accrual to account for non-compliance with ACT and loss to follow-up.

Results 919 of 926 women recruited from April 2011 to June 2017 were eligible and included in the primary analysis: 463 assigned ACT, 456 control. ACT was started in 361 (78\%) women assigned to receive it. Median follow-up was 60 months (IQR 45-65). OS at 5 years was similar in those assigned ACT versus control $(72 \%$ vs $71 \%$, difference $<1 \%$, $95 \%$ CI -6 to $+7 ; \mathrm{P}=0.91)$. The hazard ratio for OS was 0.91 , (95\% CI 0.70 to 1.18 ). PFS at 5 years was similar in those assigned ACT versus control (63\% vs 61\%, difference $2 \%, 95 \% \mathrm{CI}-5$ to $+9 ; \mathrm{P}=0.61)$. The hazard ratio for PFS was $0 \cdot 87$, (95\% CI 0.70 to 1.08$)$. AE of grade $3-5$ within a year of randomisation occurred in $81 \%$ who were assigned and received ACT versus $62 \%$ assigned control. There was no evidence of differences between treatment groups in $\mathrm{AE}$ beyond 1 year of randomisation. Patterns of disease recurrence were similar in the two treatment groups.

Conclusion Adjuvant chemotherapy given after standard cisplatin-based chemoradiation for women with locally advanced cervical cancer did not improve OS or PFS.

\section{Plenary 5: Oral Abstract Presentations}

\begin{tabular}{|l|l}
\hline $0011 / \# 287$ & PROGNOSTIC SIGNIFICANCE OF 'P53 SIGNATURE' \\
& (FIELDS OF DYSPLASIA) AND IN SITU MARGIN \\
& STATUS IN ORGAN-CONFINED HPV-INDEPENDENT \\
& P53 ABNORMAL VULVAR SQUAMOUS CELL \\
& CARCINOMA
\end{tabular}

${ }^{1}$ E Thompson*, ${ }^{2} \mathrm{~A}$ Jamieson, ${ }^{3} \mathrm{~J}$ Huvila, ${ }^{4} \mathrm{RW}-\mathrm{C}$ Wong, ${ }^{5} \mathrm{G}$ Trevisan, ${ }^{6} \mathrm{~N}$ Almadani, ${ }^{1} \mathrm{~J}$ Senz ${ }^{6} \mathrm{~J} \mathrm{Ho},{ }^{6} \mathrm{C}$ Gilks, ${ }^{2} \mathrm{~J}$ Mcalpine, ${ }^{6} \mathrm{~L}$ Hoang. ${ }^{1}$ Univeristy of British Columbia, Molecular Oncology, Vancouver, Canada; ' University of British Columbia, Gynecologic Oncology, Vancouver, Canada; ${ }^{3}$ University of Turku, Pathology, Turku, Finland; ${ }^{4}$ Pamela Youde Nethersole Eastern Hospita, Clinical Pathology, Hong Kong, Hong Kong PRC; ${ }^{5}$ Barts Health NHS Trust, Cellular Pathology, London, UK; ${ }^{6}$ UBC, Vancouver General Hospital, Pathology and Laboratory Medicine, Vancouver, Canada

\subsection{6/ijgc-2021-IGCS. 11}

Objectives Vulvar squamous cell carcinomas (VSCCs) can be stratified by HPV and TP53 mutation status to prognostically significant risk groups using p16 and p53 IHC. Treatment guidelines do not address optimal management of high molecular risk (TP53 mutated) pre-invasive neoplasia found at resection margins. Herein, we used p53 IHC to evaluate margin status in a retrospective cohort of HPV-independent (HPV-I) p53abn VSCCs.

Methods Surgically staged I-II HPV-I p53abn VSCCs from a single institution underwent margin (re)assessment using p53 IHC. Cases were segregated to i) morphologic dVIN at margin ii) vulvar skin with abnormal p53 IHC staining at margin and subtle morphologic features insufficient for dVIN iii) margins clear by morphology \& p53 IHC TP53 mutation status was evaluated by next-generation sequencing (group ii). Clinicopathologic and outcome data was collated using a standardized collection tool. 